\title{
Time-resolved study of the photodissociation of $\mathrm{Au}_{2} \mathrm{O}^{-}$
}

\author{
Kiichirou Koyasu, Marco Niemietz *, Matthias Götz, Gerd Ganteför \\ Department of Physics, University of Konstanz, D-78457 Konstanz, Germany
}

\begin{abstract}
Photodissociation of the $\mathrm{Au}_{2} \mathrm{O}^{-}$anion following absorption of a $3.1 \mathrm{eV}$ photon is studied using time-resolved photoelectron spectroscopy. A new spectral feature appearing after $1 \mathrm{ps}$ indicates the existence of a long-lived excited state with a geometry different from that of the ground state. With further increasing delay the pump-probe feature shifts to higher binding energy. At $7 \pm 2$ ps after excitation no further shift is observed, but now fragmentation into $\mathrm{AuO}^{-}+\mathrm{Au}$ and $\mathrm{Au}^{-}+\mathrm{AuO}$ starts with a time constant of $110 \pm 3$ ps. The dissociation process is accompanied by a decrease of the excited state feature. The spectra indicate that the delayed onset of fragmentation is caused by the time the system needs to reach a new minimum on the excited state potential surface. Hence, the time-resolved photoelectron spectra mirror part of the motion of the anion on the excited state potential surface.
\end{abstract}

\section{Introduction}

Interaction of photons (e.g., sun light) with matter usually just leads to heating of the surface. For photochemical applications and energy production from sun light, storage of the photon energy in an excited state and subsequent conversion into chemical energy (e.g. photodissociation of water into oxygen and hydrogen) or electrical energy (e.g. creation of an electron-hole pair in a solar cell) is desired. In both cases, the existence of a long-lived excited electronic state is essential to store the energy of the photon for further processing. Conversion of the energy of a visible photon into chemical energy could proceed via the following scheme:

$$
\mathrm{AB}+h v \rightarrow \mathrm{AB}^{*} \rightarrow \mathrm{A}+\mathrm{B}
$$

$\mathrm{AB}^{*}$ is an excited state of the molecule, which subsequently decays by dissociation. In the case of $\mathrm{H}_{2} \mathrm{O}$, the energy needed for splitting into hydrogen and oxygen is higher than the energy of a photon in the visible range. Thus,

\footnotetext{
* Corresponding author. Fax: +49 7531885133.

E-mail address: marco.niemietz@uni-konstanz.de (M. Niemietz).
}

more complex reactions, e.g. involving a catalyst particle, need to be considered [1].

Metal nanoparticles and clusters have outstanding optical properties, such as the huge cross sections for photoexcitation of Mie plasmons [2,3] and their surprising catalytic activities [4]. However, in many cases photoabsorption results in ultrafast thermalization mediated by two mechanisms: (i) electron-electron interactions distribute the energy among the delocalized metal electrons [5] and (ii) internal conversion transfers the energy of electronic excitations directly into vibrational excitations (phonons) $[6,7]$. The first mechanism requires a sufficiently high density of states above the Fermi level (or highest occupied molecular orbital in molecules and clusters) [5]. The latter process dominates in species with a rather flexible geometry [8]. In small clusters with a lower density of states and a rather rigid geometric structure, the situation is different: long-lived excited states $[9,10]$ and direct photofragmentation [10] exist, making these nanoparticles promising candidates for photochemistry.

In recent years, especially $\mathrm{Au}$ clusters have gained considerable attention owing to their extraordinary catalytic properties $[4,11,12]$. Additionally, long-lived excited states have been discovered for some gold clusters $[9,10]$. 
Encouraged by these findings, we started a systematic survey of the photochemical properties of $\mathrm{Au}_{n} \mathrm{O}_{m}^{-}, \mathrm{Au}_{n} \mathrm{H}_{m}^{-}$and $\mathrm{Au}_{n}\left(\mathrm{H}_{2} \mathrm{O}\right)_{m}^{-}$clusters, searching for promising candidates for applications in catalysis and energy conversion.

In the present work, first results on $\mathrm{Au}_{2} \mathrm{O}^{-}$are presented, which is one of the simplest specimen among the clusters listed above. $\mathrm{Au}_{2} \mathrm{O}^{-}$is studied using the well-established method of time-resolved photoelectron spectroscopy (TR-PES), which is suitable for observation of the time evolution of a system in real time [13-16]. The clusters are negatively charged, being on the one hand a disadvantage, since information about the properties of the anion is gained, but on the other hand allowing for accurate mass selection. From results on the negatively charged clusters, properties of neutral clusters can be inferred. In addition, on certain surfaces and on fuel cell electrodes, catalyst particles can be negatively charged, allowing a direct comparison with our gas phase data.

The $\mathrm{Au}_{2} \mathrm{O}^{-}$anions are excited by $3.1 \mathrm{eV}$ photons onto the excited state potential surface and progress towards a local minimum. After $7 \pm 2$ ps the anion reaches the local minimum and fragmentation starts with a time constant of $110 \pm 30$ ps:

$$
\begin{aligned}
& \mathrm{Au}_{2} \mathrm{O}^{-}+h v \rightarrow \mathrm{Au}_{2} \mathrm{O}^{-*} \rightarrow \mathrm{AuO}^{-}+\mathrm{Au} \\
& \mathrm{Au}_{2} \mathrm{O}^{-}+h v \rightarrow \mathrm{Au}_{2} \mathrm{O}^{-*} \rightarrow \mathrm{Au}^{-}+\mathrm{AuO}
\end{aligned}
$$

In case of this molecule, TR-PES is able to monitor part of the path of the system propagating on the excited state surface. This is an example of the energy of a visible photon converted into chemical energy by a long-lived excited state, in contrast to fast thermalization observed for many other clusters $[17,18]$. Further studies of larger clusters are on the way.

\section{Experimental setup}

The experimental setup has been described in detail elsewhere [19]. Gold cluster anions are generated in a pulsed arc cluster ion source (PACIS), and $\mathrm{O}_{2}$ is introduced into the source to generate $\mathrm{Au}_{\mathrm{n}} \mathrm{O}_{\mathrm{m}}^{-}$clusters. The cluster anions are mass-selected using a reflectron type time-of-flight mass spectrometer. The bunch of $\mathrm{Au}_{2} \mathrm{O}^{-}$anions is irradiated by two laser pulses (pump \& probe) with variable time delay, both pulses being the second harmonic of a Ti:sapphire femtosecond (fs) laser system $(400 \mathrm{~nm}=3.1 \mathrm{eV})$. The time-resolution of the setup is $110 \mathrm{fs}$ [8]. The kinetic energy of the detached electrons is measured using a 'magnetic bottle' type time-of-flight electron spectrometer, operated at a moderate energy resolution of $100 \mathrm{meV}$. The temperature of the cluster anions is estimated to be roughly room temperature, since the anions spend a sufficient amount of time inside the water-cooled extender $(4 \mathrm{~mm}$ diameter, $20 \mathrm{~cm}$ long), to allow for thermalization with the extender walls. Great care has been taken to optimize the pulse energies of both pulses. The energies of both pulses (pump \& probe) are optimized at long delays, maximizing the photo- fragment signal and minimizing the two-photon signal from each individual pulse. As a result, the pulse energy of the pump pulse is higher than the one of the probe pulse and there is a considerable two-photon signal generated by the pump alone, which does not depend on the delay between both pulses. The pulse energy of the probe pulse is set to a lower value, allowing effective one-photon detachment from the excited $\mathrm{Au}_{2} \mathrm{O}^{-*}$ species and the charged photofragments $\mathrm{Au}^{-}$and $\mathrm{AuO}^{-}$. From the neutral fragments no signal can be detected due to their high ionization potentials.

\section{Results}

Fig. 1 displays ground state photoelectron spectra of those anions $\left(\mathrm{Au}^{-}, \mathrm{AuO}^{-}, \mathrm{Au}_{2}^{-}\right.$, and $\left.\mathrm{Au}_{2} \mathrm{O}^{-}\right)$, which might play a role in the photofragmentation of $\mathrm{Au}_{2} \mathrm{O}^{-}$. All spectra in Fig. 1 are recorded with a single pulse of the femtosecond laser $(3.1 \mathrm{eV}$ or $4.65 \mathrm{eV})$. The spectra of $\mathrm{Au}^{-}$and $\mathrm{AuO}^{-}$are measured using the second harmonic of the Ti:sapphire laser $(3.1 \mathrm{eV})$, while the spectra of $\mathrm{Au}_{2}^{-}$and $\mathrm{Au}_{2} \mathrm{O}^{-}$are obtained with the third harmonic $(4.65 \mathrm{eV})$. In the observable energy range the spectrum of $\mathrm{Au}^{-}$exhibits a single peak at $2.3 \mathrm{eV}$ binding energy (marked A), corresponding to the electron affinity of $\mathrm{Au}$ [20]. For

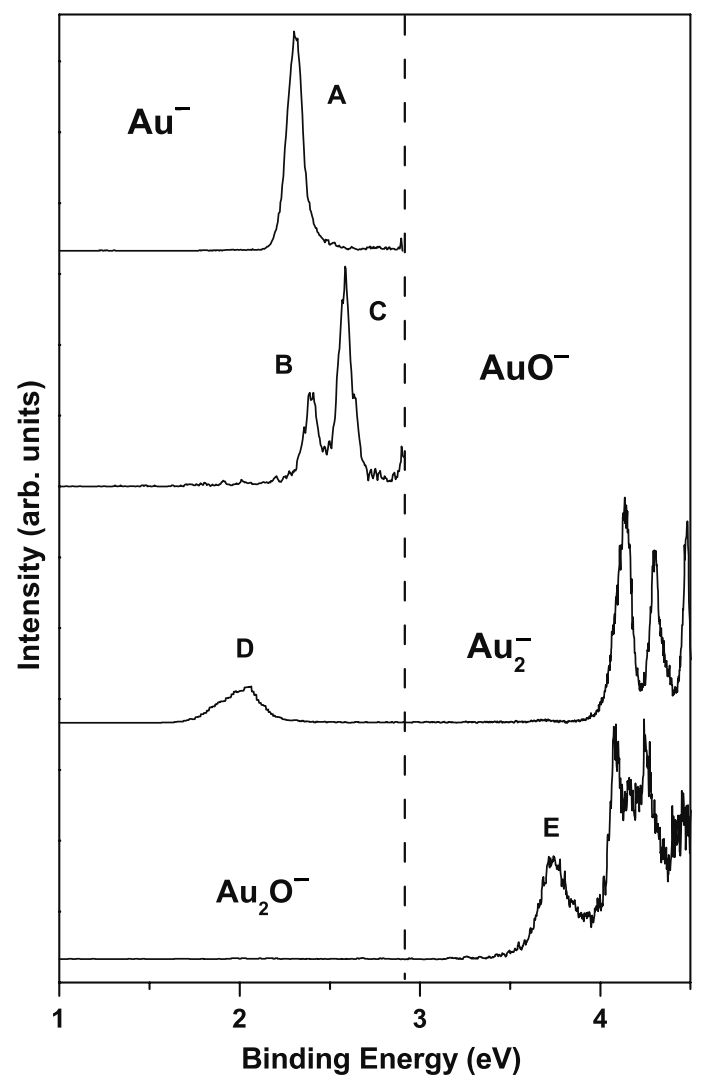

Fig. 1. Photoelectron spectra of $\mathrm{Au}^{-}, \mathrm{AuO}^{-}, \mathrm{Au}_{2}^{-}$and $\mathrm{Au}_{2} \mathrm{O}^{-}$recorded with a single pulse of the femtosecond laser system. The pulse energy is reduced below the onset of two-photon processes. The upper two spectra are recorded using a photon energy of $3.1 \mathrm{eV}$, whereas for the lower two $4.65 \mathrm{eV}$ was used. 
$\mathrm{AuO}^{-}$, two peaks at $2.4 \mathrm{eV}$ and $2.6 \mathrm{eV}$ (marked $\mathrm{B}$ and $\mathrm{C}$ ) are observed with the feature at $2.4 \mathrm{eV}$ having about $44 \%$ of the intensity of the $2.6 \mathrm{eV}$ peak. Both spectra agree with earlier measurements $[20,21]$. In the energy regime accessible by the $3.1 \mathrm{eV}$ pump photons, $\mathrm{Au}_{2}^{-}$exhibits a single feature centered at $2.0 \mathrm{eV}$ (marked D), while for $\mathrm{Au}_{2} \mathrm{O}^{-}$the vertical detachment energy is higher than $3.1 \mathrm{eV}$ (marked E), thus no one-photon signal appears in the pump-probe spectra. Again, the spectra agree with earlier data recorded with standard pulsed lasers $[20,22]$.

The high electron affinity of $\mathrm{Au}_{2} \mathrm{O}^{-}$makes it an ideal candidate for photoexcitation experiments, since the competing channel of photodetachment is closed for photon energies up to $3.5 \mathrm{eV}$. Species with such a high EA allow for the observation of the time evolution of high-lying excited states

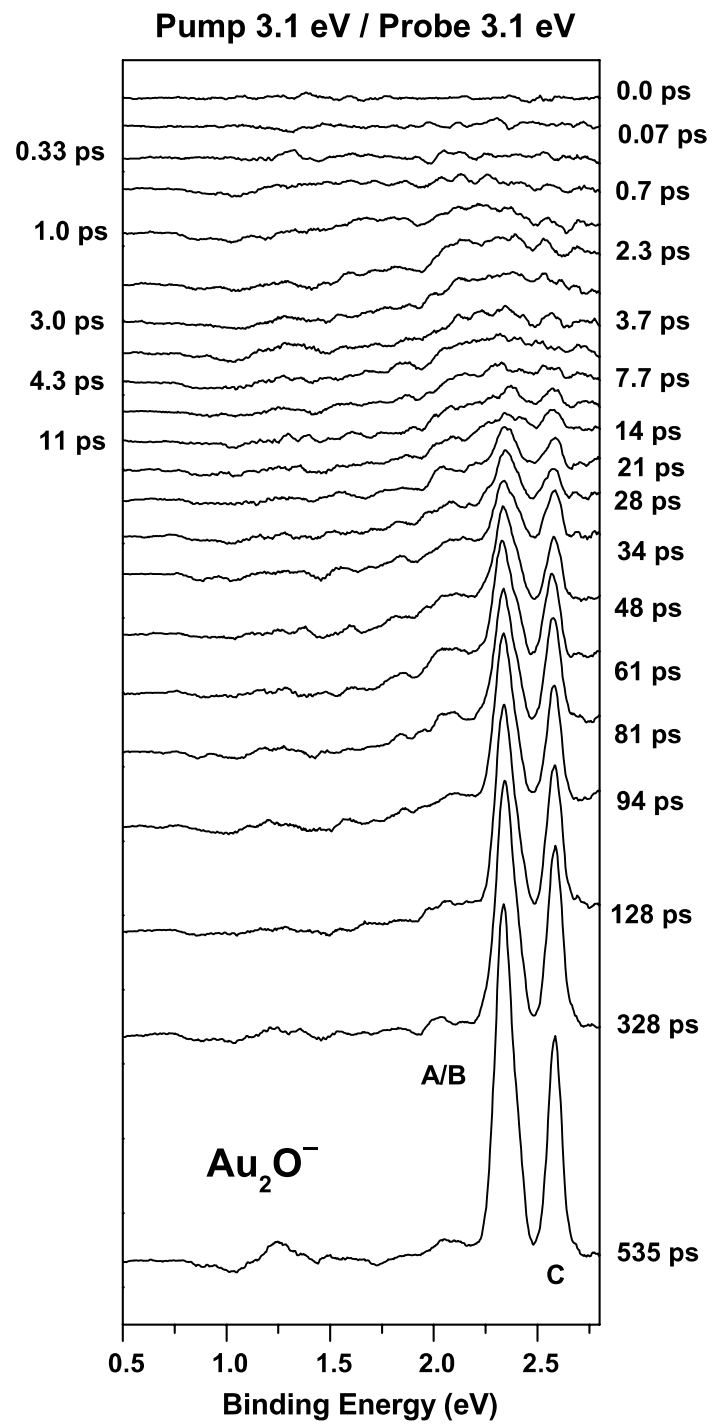

Fig. 2. Series of pump-probe photoelectron spectra of $\mathrm{Au}_{2} \mathrm{O}^{-}$. Both pulses have a photon energy of $3.1 \mathrm{eV}$. The delay between pump and probe pulse is given in picoseconds for each trace. The pulse energy of the pump is above the threshold of two-photon processes, while the one of the probe is kept below. Only the delay dependent signal is displayed, the delay independent signal has been subtracted. The two peaks marked A/B and C are assigned to the photofragments $\mathrm{Au}^{-}$and $\mathrm{AuO}^{-}$, respectively (for further information refer to the text). in a TR-PES experiment. In addition, for $\mathrm{Au}_{2} \mathrm{O}^{-}$all possible charged photofragments can be observed using $3.1 \mathrm{eV}$ photons due to their low electron affinities.

Fig. 2 displays a series of pump-probe photoelectron spectra of $\mathrm{Au}_{2} \mathrm{O}^{-}$. The spectra are difference spectra, showing the delay dependent contribution only. At zero delay, the difference signal vanishes. In the spectra starting at $1.0 \mathrm{ps}$ delay, a broad maximum between $2.0 \mathrm{eV}$ and $2.5 \mathrm{eV}$ appears. With further increasing delay, two peaks on top of this feature grow at binding energies of $2.35 \mathrm{eV}$ (marked $\mathrm{A} / \mathrm{B}$ ) and $2.6 \mathrm{eV}$ (marked $\mathrm{C}$ ), while the intensity of the broad maximum decreases simultaneously.

Using $3.1 \mathrm{eV}$ laser pulses, three basically different spectra can be recorded for $\mathrm{Au}_{2} \mathrm{O}^{-}$. At zero delay, the signal is dominated by the two-photon contribution of the (more intense) pump pulse. It is assigned to the spectrum of the excited state of $\mathrm{Au}_{2} \mathrm{O}^{-}$in the geometry of the anion ground state (Fig. 3, lower trace, left part). The spectrum is delayindependent and does not appear in Fig. 2. The excited

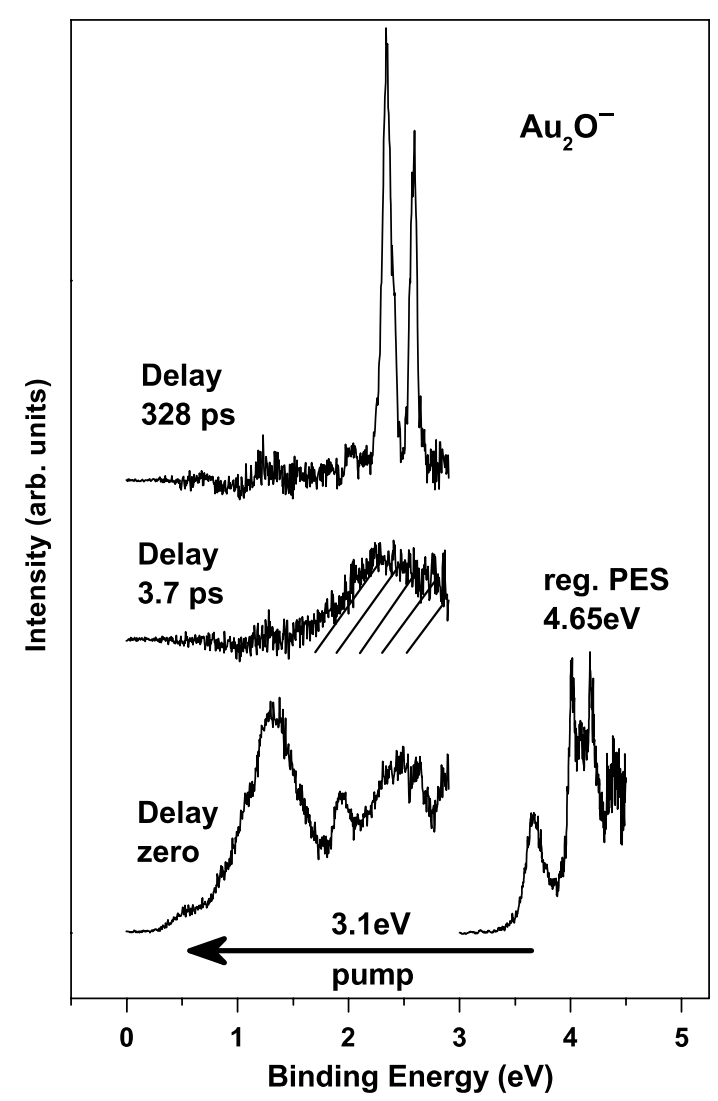

Fig. 3. Selected photoelectron spectra of $\mathrm{Au}_{2} \mathrm{O}^{-}$: The lowest trace displays a comparison between the spectrum of the ground state recorded with $4.65 \mathrm{eV}$ photon energy (bottom right, probe only) and the spectrum obtained with $3.1 \mathrm{eV}$ (bottom left, pump only). The pulse energy of the $3.1 \mathrm{eV}$ pump pulse is above the threshold of two-photon processes. This two-photon signal is delay-independent and subtracted from each spectrum displayed in Fig. 2. The middle trace shows a difference spectrum recorded at a delay of $3.7 \mathrm{ps}$ before onset of fragmentation. This spectrum is assigned to the excited state of $\mathrm{Au}_{2} \mathrm{O}^{-}$in a new geometry. The upper trace displays a difference spectrum at long delay ( $328 \mathrm{ps}$ ). The intensity of the broad maximum observed at $3.7 \mathrm{ps}$ is reduced, and the spectrum is dominated by the features assigned to the photofragments. 
state develops with increasing time into a new geometry, yielding a broad maximum, which dominates the pumpprobe difference spectra at a delay of 3.7 ps (Fig. 3, middle trace). At further increasing time delay, the broad maximum disappears and features assigned to photofragments are observed in the pump-probe difference spectrum (Fig. 3, upper trace).

\section{Discussion}

Both peaks visible at long delays (Fig. 2, lowest trace, marked $\mathrm{A} / \mathrm{B}$ and $\mathrm{C}$ ) are assigned to the anionic photofragments $\mathrm{Au}^{-}$and $\mathrm{AuO}^{-}$. The electron affinities of $\mathrm{Au}$ and $\mathrm{AuO}$ are the same within $0.065 \mathrm{eV}[20,21]$. Accordingly, the feature at lower binding energy (marked A/B) consists of two components, a contribution from the low binding energy feature of $\mathrm{AuO}^{-}$(Fig. 1, peak B) and the signal from the $\mathrm{Au}^{-}$anion (Fig. 1, peak $\mathrm{A}$ ).

The time constants of both dissociation channels, yielding $\mathrm{Au}^{-}$or $\mathrm{AuO}^{-}$, are determined by plotting their photofragment intensities versus delay time. The intensity of $\mathrm{AuO}^{-}$is directly proportional to that of peak $\mathrm{C}$ in Fig. 2. In case of $\mathrm{Au}^{-}$, the intensity corresponds to that of peak $\mathrm{A} / \mathrm{B}$, corrected by the contribution from $\mathrm{AuO}^{-}$. This has been done by subtracting $44 \%$ of the intensity of feature $C$, since this reflects the contribution of peak $B$ in feature A/B, as can be seen in the second trace of Fig. 1. Assuming a single exponential growth, the intensity for each dissociation channel is given by

$I=I_{\max }\left(1-\exp ^{-t / \tau}\right)$,

where $\tau$ is the time constant for fragmentation and $I_{\max }$ corresponds to the maximum fragment ion intensity, depending on the energies of pump and probe and on the cross sections for photoexcitation of the parent anion and photodetachment of both fragment anions. Both parameters $\left(I_{\mathrm{m}-}\right.$ ax,$\tau)$ can be different for the two channels yielding $\mathrm{Au}^{-}$and $\mathrm{AuO}^{-}$. In addition, the delay of the onset for fragmentation might be different. To extract the time constant $\tau$ and the onset delay, it is convenient to plot $\left(I_{\max }-I\right) / I_{\max }$ on a logarithmic scale versus $t$ (Fig. 4). Now, fragmentation obeying a single exponential law appears as linear increase starting at 1 with a slope corresponding to $\tau$. Within the error bars of our experiment, both fragmentation channels can be described by the same time constant $(110 \pm 30 \mathrm{ps})$ and the same onset $(7 \pm 2 \mathrm{ps})$. Hence, the fragmentation mechanism is very similar for both channels.

In order to shed light on the possible fragmentation channels, it is useful to evaluate the energies involved into the possible decay paths:

$$
\begin{aligned}
\mathrm{Au}_{2} \mathrm{O}^{-*} & \rightarrow \mathrm{AuO}^{-}+\mathrm{Au} \\
& \rightarrow \mathrm{Au}^{-}+\mathrm{AuO} \\
& \rightarrow \mathrm{Au}_{2}^{-}+\mathrm{O} \\
& \rightarrow \mathrm{O}^{-}+\mathrm{Au}_{2}
\end{aligned}
$$

The excitation energy necessary for process III is predicted from theory [23] to be $3.14 \mathrm{eV}$, which is slightly higher than

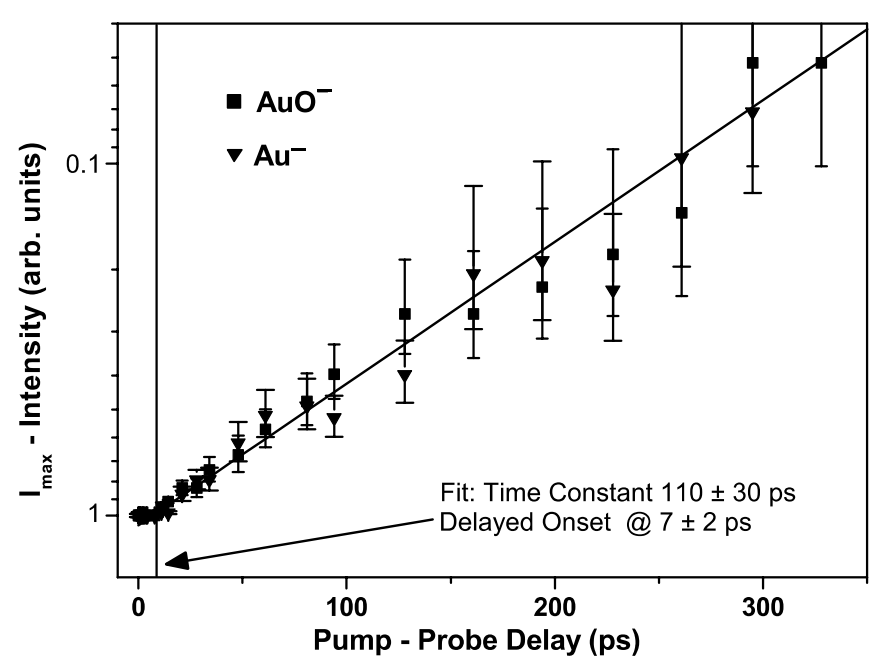

Fig. 4. Delay dependence of the intensities of the photofragments, as extracted from the series of pump-probe spectra displayed in Fig. 2. Displayed is the difference (normalized to 1) between the extrapolated maximum intensity $I_{\max }$ at infinite delay and the measured intensity $I$. Both signals start to grow with a delay of $7 \pm 2$ ps. Within the accuracy of the experiment both fragment peaks increase exponentially with a time constant of $110 \pm 30 \mathrm{ps}$.

the excitation energy of our system. This finding agrees with the fact that we do not observe a signal from $\mathrm{Au}_{2}^{-}$. Process IV requires even higher energy due to the low electron affinity of $\mathrm{O}$ and can also be excluded. Processes I and II might be very close in energy, because the electron affinities of $\mathrm{Au}$ and $\mathrm{AuO}$ are similar $[20,21]$. This fact may explain that both processes are observed with comparable probability.

Delayed onsets of the photofragment signal have been observed in similar experiments earlier [24,25]. Their origin is still unclear, but two mechanisms can be considered as possible explanations:

1. The system needs time to evolve on the excited state potential energy surface until it approaches a minimum, from which a decay is possible (e.g., via a conical intersection).

2. The photofragments need time to separate after relaxation into the ground state.

With our experimental method it is possible to examine the excited anion in detail during the first few picoseconds after excitation (Fig. 5). The data are the same as the ones displayed in Fig. 2, but smoothed and plotted with expanded intensity scale. Within 1 ps after the excitation a broad maximum appears, which further grows in intensity and shifts towards higher binding energy. After about 7 ps no further change of shape and position can be observed. Fig. 5 suggests the following interpretation: the maximum corresponds to the anion in a local minimum of the excited state potential surface. Its equilibrium geometry is different from the one the anionic ground state and the change of shape and position of the broad feature reflects the geometric rearrangement which the system undergoes after excitation. As long as the geometry of the 
Pump 3.1 eV / Probe $3.1 \mathrm{eV}$

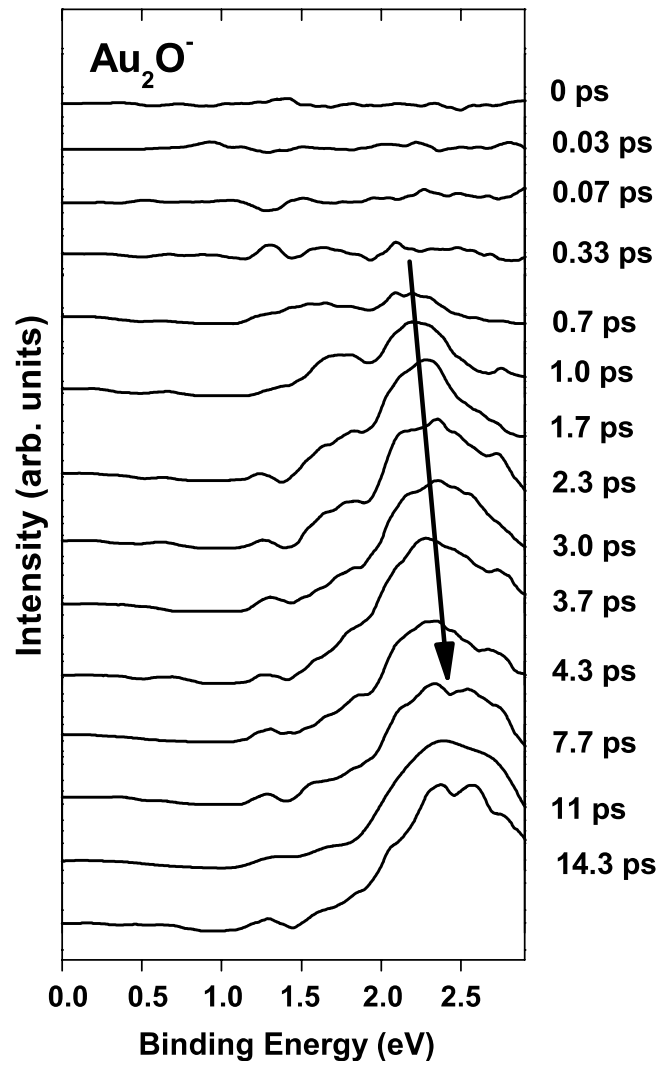

Fig. 5. Details of the dynamics of $\mathrm{Au}_{2} \mathrm{O}^{-}$for short delay times corresponding to an expanded view of the data in Fig. 2. After roughly $1 \mathrm{ps}$ a maximum appears in the high binding energy regime accompanied by a dip at low binding energy as a result of the subtraction procedure. With increasing delay, the maximum shifts slightly towards higher binding energy (arrow).

excited anion is very similar to the initial geometry, the difference spectra displayed in Figs. 2 and 5 show a vanishing signal. With increasing geometric rearrangement a gradually increasing peak appears, shifting in position and shape until the anion approaches the new equilibrium geometry. Hence, the data strongly support the first mechanism mentioned above being responsible for the delayed onset.

\section{Conclusion}

Photofragmentation of $\mathrm{Au}_{2} \mathrm{O}^{-}$induced by excitation with a $3.1 \mathrm{eV}$ photon is studied using TR-PES. The data indicate the existence of an excited state of the anion with a rather long lifetime of more than hundred picoseconds. From this excited state, the parent anion decays into $\mathrm{Au}^{-}+\mathrm{AuO}$ or $\mathrm{AuO}^{-}+\mathrm{Au}$. Fragmentation does not start immediately after excitation but with a considerable delay of $7 \pm 2$ ps. A detailed study of the spectra at short delay times indicates that the delayed onset for fragmentation corresponds to the time the system needs to approach a local minimum on the excited state potential surface.

The data presented on $\mathrm{Au}_{2} \mathrm{O}^{-}$are part of a systematic study of the photochemical properties of $\mathrm{Au}_{n} \mathrm{O}_{m}^{-}, \mathrm{Au}_{n} \mathrm{H}_{m}^{-}$ and $\mathrm{Au}_{n}\left(\mathrm{H}_{2} \mathrm{O}\right)_{m}^{-}$clusters, which aims in finding promising candidates for photocatalysis and energy conversion. This first result on $\mathrm{Au}_{2} \mathrm{O}^{-}$reinforces the decision to focus on $\mathrm{Au}$ clusters, since, in contrast to studies on other reacted metal clusters, a long-lived excited state has been found and fast thermalization is not the dominant decay channel.

\section{Acknowledgements}

We acknowledge DFG (Deutsche Forschungsgemeinschaft) and CAP (Center for Applied Photonics, University of Konstanz) for the financial support. One of the authors (K.K.) is grateful to JSPS Postdoctoral Fellowships for Research Abroad (2006-2008) for financial support.

\section{References}

[1] K. Maeda, K. Teramura, D. Lu, T. Takata, N. Saito, Y. Inoue, K. Domen, Nature 440 (2006) 295.

[2] K. Selby, M. Vollmer, J. Masui, V. Kresin, W.A. de Heer, W.D. Knight, Phys. Rev. B 40 (1989) 5417.

[3] J. Tiggesbäumker, L. Köller, K.-H. Meiwes-Broer, A. Liebsch, Phys. Rev. A 48 (1993) R1749.

[4] M. Haruta, Catal. Today 36 (1997) 153.

[5] C. Frischkorn, M. Wolf, Chem. Rev. 106 (2006) 4207.

[6] J. Michl, V. Bonacic-Koutecky, Electronic Aspects of Organic Photochemistry, Wiley, New York, 1990.

[7] M. Klessinger, J. Michl, Excited States and Photochemistry of Organic Molecules, VCH, New York, 1995.

[8] M. Niemietz, M. Engelke, Y.D. Kim, G. Ganteför, Phys. Rev. B 75 (2007) 085438.

[9] M. Niemietz, P. Gerhardt, G. Ganteför, Y.D. Kim, Chem. Phys. Lett. 380 (2003) 99 .

[10] G. Ganteför, S. Kraus, W. Eberhardt, J. Elec. Spec. Relat. Phenom. 88-91 (1997) 35.

[11] M. Valden, X. Lai, D.W. Goodman, Science 281 (1998) 1647.

[12] B. Yoon et al., Science 307 (2005) 403.

[13] M.T. Zanni, B.J. Greenblatt, A.V. Davis, D.M. Neumark, J. Chem. Phys. 111 (1999) 2991.

[14] J.R.R. Verlet, A.E. Bragg, A. Kammrath, O. Cheshnovsky, D.M. Neumark, J. Chem. Phys. 121 (2004) 10015.

[15] N. Pontius, P.S. Bechthold, M. Neeb, W. Eberhardt, Phys. Rev. Lett. 84 (2000) 1132.

[16] N. Pontius, M. Neeb, W. Eberhardt, G. Lüttgens, P.S. Bechthold, Phys. Rev. B 67 (2003) 035425.

[17] G. Lüttgens, N. Pontius, P.S. Bechthold, M. Neeb, W. Eberhardt, Phys. Rev. Lett. 88 (2002) 076102.

[18] M. Neeb, J. Stanzel, N. Pontius, W. Eberhardt, G. Lüttgens, P.S Bechthold, C. Friedrich, J. Elec. Spec. Relat. Phenom. 144-147 (2005) 91.

[19] P. Gerhardt, M. Niemietz, Young Dok Kim, G. Ganteför, Chem. Phys. Lett. 382 (2003) 454.

[20] J. Ho, K. Ervin, W.C. Lineberger, J. Chem. Phys. 93 (1990) 6987.

[21] T. Ichino, A.J. Gianola, D.H. Andrews, W.C. Lineberger, J. Phys. Chem. A 108 (2004) 11307.

[22] Q. Sun, P. Jena, Y.D. Kim, M. Fischer, G. Ganteför, J. Chem. Phys. 120 (2004) 6510.

[23] M.L. Kimble, N.A. Moore, G.E. Johnson, A.W. Castleman Jr., C. Bürgel, R. Mitrić, V. Bonačić-Koutecký, J. Chem. Phys. 125 (2006) 204311.

[24] V. Dribinski, J. Barbera, J.P. Martin, A. Svendsen, M.A. Thompson, R. Parson, W.C. Lineberger, J. Chem. Phys. 125 (2004) 133405.

[25] F. Muntean, M.S. Taylor, A.B. McCoy, W.C. Lineberger, J. Chem. Phys. 121 (2004) 5676. 